\title{
Current understandings and prospects of antibody-drug conjugates (ADCs) for the treatment of breast cancer: a narrative review
}

\author{
Chunfang Hao ${ }^{1}$, Hong Liu $^{2}$ \\ ${ }^{1}$ Department of Breast Oncology, Tianjin Medical University Cancer Institute \& Hospital, National Clinical Research Center for Cancer, Key \\ Laboratory of Cancer Prevention and Therapy, Tianjin's Clinical Research Center for Cancer, Tianjin, China; ${ }^{2}$ The Second Surgical Department of \\ Breast Cancer, Tianjin Medical University Cancer Institute \& Hospital, National Clinical Research Center for Cancer, Key Laboratory of Cancer \\ Prevention and Therapy, Tianjin's Clinical Research Center for Cancer, Tianjin, China \\ Contributions: (I) Conception and design: Both authors; (II) Administrative support: Both authors; (III) Provision of study materials or patients: \\ Both authors; (IV) Collection and assembly of data: Both authors; (V) Data analysis and interpretation: Both authors; (VI) Manuscript writing: Both \\ authors; (VII) Final approval of manuscript: Both authors. \\ Correspondence to: Chunfang Hao. Department of Breast Oncology, Tianjin Medical University Cancer Institute \& Hospital, National Clinical \\ Research Center for Cancer, Key Laboratory of Cancer Prevention and Therapy, Tianjin's Clinical Research Center for Cancer, Tianjin 300060, \\ China. Email: haochf@163.com.
}

Objective: To provide a review of the advances of antibody-drug conjugates (ADCs) for the treatment of breast cancer.

Background: The recent successes and approval of ADCs for hematologic malignancies and solid malignant tumor including breast cancer have transformed cancer therapy. By May 2021, 10 different ADCs have been approved by the USA FDA, among which three ADCs have been approved in breast cancer. Remarkably, the phenomenon of numerus successes with the next-generation ADCs in breast cancer, offers the prospect of completely shifting the paradigm of breast cancer treatment. Most anticipated is that these breakthroughs would ultimately translate to the benefit of patients.

Methods: A literature search was conducted for up-to-date publications and ongoing clinical trials of $\mathrm{ADCs}$ and breast cancer, and then the review report was developed. In the beginning of the report, a brief introduction of $\mathrm{ADC}$ was given in the perspective of structure and mechanism of action. Following which, the great advances of three approved ADCs were evoked, and the important value of these clinical trials was highlighted. Meanwhile, a list of several promising ADCs targeting human epidermal growth factor receptor 2 (HER2), trophoblast cell-surface antigen 2 (TROP-2) and beyond that have been under development for breast cancer therapy were provided. Finally, it is expected that these ADCs will enlighten the future of breast cancer treatment.

Conclusions: Herein, we present a brief overview of ADCs for breast cancer, with the understanding that novel ADCs will demonstrate great clinical value over the next few years.

Keywords: Human epidermal growth factor receptor 2 (HER2); trastuzumab emtansine (T-DM1); antibody-drug conjugate (ADC); sacituzumab govitecan; trastuzumab deruxtecan

Received: 15 September 2021; Accepted: 30 October 2021; Published: 31 October 2021.

doi: $10.21037 /$ tbcr-21-30

View this article at: https://dx.doi.org/10.21037/tbcr-21-30 


\section{A brief introduction of antibody-drug conjugates (ADCs): structure and mechanism of action}

ADCs consist of a highly specific recombinant monoclonal antibody $(\mathrm{mAb})$ that recognizes tumor-associated antigens and is covalently conjugated to a high potency cytotoxic drug via stable chemical linkage through a synthetic linker. Though targeted antibody-delivered cytotoxic payload is based on a century-old concept of "magic bullet" by German chemist Paul Ehrlich (1), with the first human trial of ADC in the 1980s (2), only until recently, advances in antibody engineering, linker optimization chemistries, and conjugation strategies (3) have improved ADC design and paved the way for transformative cancer therapy with potent and safer ADCs.

ADCs are complex biochemical platforms used in cancer treatment and exhibit unique mechanisms of action (4). After administration, ADCs may be transported in the systemic circulation as three components: naked antibody, free payload, and intact conjugate due to incomplete conjugation with different production processes and conjugation technologies. Meanwhile, ADCs may have different drug-antibody ratios (DARs). The homogeneity of ADCs is the precondition for limiting toxicity and improving the therapeutic window (5). As ADCs approach tumor site, some payload might be released prematurely into the tumor microenvironment before antibody-antigen binding, leading to some ADCs to exhibit their efficacy via slow-release chemotherapy $(6,7)$. In addition, the antibody component of some ADCs retains its activity profile post conjugation and continue to inhibit target function, block downstream signaling and/or generate immunerelated effects. For example, in preclinical studies, human epidermal growth factor receptor 2 (HER2)-targeted ADCs, trastuzumab emtansine (T-DM1) and trastuzumab deruxtecan (T-DXd), retain the function of trastuzumab, and thus can exhibit their potency via inhibiting HER2 signaling and mediating antibody-dependent cell-mediated cytotoxicity (ADCC) $(8,9)$. Following antigen binding, most ADCs are internalized via endocytosis. Once inside the lysosomes or endosomes, the payloads are released from their antibody carriers by acidolysis, enzymatic hydrolysis or reduction, following which the payloads act on their target substrates, finally resulting in cell death. Membrane permeable payloads can also diffuse through cell membranes into the tumor microenvironment, thus killing neighboring cells irrespective of target antigen expression, a phenomenon known as "bystander killing effect (10)".
We present the following article in accordance with the Narrative Review reporting checklist (available at https:// dx.doi.org/10.21037/tbcr-21-30).

\section{Approved anti-HER2 ADCs and anti-trophoblast cell-surface antigen 2 (TROP-2) ADCs in breast cancer}

HER2 is a receptor tyrosine-protein kinase and a member of the epidermal growth factor receptor (EGFR) family. It is overexpressed or amplified in approximately $15 \%$ to $20 \%$ of metastatic breast cancer (MBC) patients (11-13), which predicts an adverse outcome (13). Trastuzumab is a humanized $\mathrm{mAb}$ targeting the extracellular domain of HER2 and as the first approved anti-HER2 drug, has become the backbone of HER2 positive breast cancer management, from neoadjuvant/adjuvant in the early setting for cure to first-line treatment and beyond in the metastatic setting for rescue. Numerous other antiHER2 drugs have since been approved, including antiHER2 $\mathrm{mAb}$ such as pertuzumab and margetuximab, and small molecule tyrosine kinase inhibitors (TKIs) including lapatinib, neratinib, pyrotinib and tucatinib. T-DM1 is the first approved anti-HER2 ADC, initially as secondline treatment in HER2 positive MBC and subsequently as adjuvant treatment of HER2 positive early breast cancer (EBC) with residual invasive disease after neoadjuvant antiHER2 regimen. T-DXd became the second approved antiHER2 ADC in 2019 (14), with impressive data from the Phase II single arm Destiny-Breast01 trial, promising to change the landscape of breast cancer treatment. TROP2 , a transmembrane calcium signal transducer that is highly expressed in multiple tumor types, including breast cancer $(>90 \%)$, is identified as an ideal antigen allowing for targeted delivery by ADCs (15). In 2020, sacituzumab govitecan (SG), the first anti-TROP-2 ADC, was approved for later line therapy in metastatic triple negative breast cancer, enlarging the population of breast cancer patients treatable with ADCs. The successful story of ADCs in breast cancer has driven rapid changes for breast cancer treatment and ignited the enthusiasm of ADC research and development. The number of novel ADCs in clinical studies has dramatically increased over the past few years.

\section{T-DM1}

T-DM1 (Kadcyla) has been one of the most successful $\mathrm{ADCs}$ so far. It consists of a trastuzumab mAb to which the 
maytansinoid DM1, a microtubule inhibitory molecule, is conjugated via a non-cleavable thioether linker attached to random lysines with an average DAR of 3.5:1 (16). Similar to trastuzumab, T-DM1 inhibits HER2 receptor signaling, mediates ADCC and suppresses shedding of the HER2 extracellular domain in human breast cancer cells overexpressing HER2. The reported and ongoing clinical trials of T-DM1 were listed in Table 1.

As early as in 2012, the Phase III EMILIA (17) trial demonstrated that T-DM1 significantly prolonged progression-free survival (PFS) and overall survival (OS) than lapatinib plus capecitabine in HER2 positive advanced breast cancer patients previously treated with trastuzumab and a taxane. A subsequent phase III TH3RESA trial demonstrated that T-DM1 significantly pronged the median PFS of progressive HER2 positive advanced breast cancer patients who had received two or more HER2directed regimens in the advanced setting. T-DM1 has since become the standard second line treatment for HER2 positive MBC (18). A few years later, the phase III, openlabel KATHERINE trial showed that T-DM1 achieved a significant higher invasive disease-free survival (iDFS) rate versus trastuzumab (19) in HER2 positive EBC patients with residual invasive disease at surgery after trastuzumab and taxane-based neoadjuvant therapy. These results, for the first time, indicated that treatment escalation in the adjuvant setting with T-DM1 could become a new standard of care for HER2 positive EBC with residual invasive disease after completion of neoadjuvant therapy.

However, the development of T-DM1 as a first-line treatment for HER2 positive MBC or as a neoadjuvant/ adjuvant treatment has not been satisfactory hitherto (20-22). In several phase III trials, T-DM1-based regimens failed to show superior efficacy when compared with standard of care with trastuzumab $(\mathrm{H})$ or trastuzumab + pertuzumab $(\mathrm{H}+\mathrm{P})$, among treatment native patients in early lines. As shown in the conclusion from the trials above, T-DM1 seems to become the "back-up" option to trastuzumab-based treatment. The heterogeneous and low DAR of T-DM1 could impact its pharmacokinetics $(\mathrm{PK})$ profile and limit its therapeutic window $(5,8)$. Though T-DM1 retains the function of trastuzumab and shows great potency in preclinical studies, the clinical recommended dose $(3.6 \mathrm{mg} / \mathrm{kg})$ is notably lower than routine trastuzumab dosage $(6 \mathrm{mg} / \mathrm{kg})$. T-DM1 is designed to be stable in systemic circulation with a stable non-cleavable linker. The intracellular active metabolite of T-DM1, smcc-DM1, is a membrane impermeable payload; thus, T-DM1 has no bystander effect, which may limit its efficacy compared with trastuzumab plus chemotherapy $(10,16)$.

Theoretically, combining T-DM1 with other treatment could further boost its efficacy. The phase II TEAL study is conducted comparing the combination regimen of T-DM1, lapatinib, and nab-paclitaxel (KLT) with the standard treatment of trastuzumab, pertuzumab, and paclitaxel (PHT) as neoadjuvant treatment in HER2 positive EBC (23). The small size trial surprisingly showed KTL regimen had higher residual cancer burden (RCB) 0 or I than PHT $(100 \%$ vs. $62.5 \%, \mathrm{P}=0.0035)$. The combination treatment of T-DM1 with tucatinib and atezolizumab has under evaluation in several phase III trials (NCT03975647, NCT04740918, NCT04873362 and NCT04457596).

Interestingly, de-escalation ATEMPT (TBCRC-033 trial) has been carried to determine if T-DM1 could cause less toxicity than paclitaxel plus trastuzumab (TH) and yielded clinically acceptable benefit among patients with stage I HER2 positive EBC (24). However, the incidence of clinically relevant toxicities was comparable between T-DM1 and HT arm (46\% vs. 47\%, $\mathrm{P}=0.83$ ), while the amenorrhea was less likely in recipients of T-DM1 than TH (25). The 3-year iDFS for T-DM1 was 97.8\% (95\% CI: $96.3 \%$ to $99.3 \%$ ), while a descriptive analysis of $\mathrm{TH}$ treatment showed an iDFS rate of $93.4 \%$ (95\% CI: $88.7 \%$ to $98.2 \%$ ). Considering this trial included $75 \%$ hormonal receptor positive patients with low event rate, longer followup is needed to obtain more meaningful DFS data (24). More attractively, the ATEMPT 2.0 trial (NCT04893109) is evaluating a more optimized regimen in this setting ( $\mathrm{T}$ DM1 $\times 6$ cycles $\rightarrow$ trastuzumab subcutaneous $\times 11$ cycles $)$.

\section{Trastuzumab deruxtecan}

Trastuzumab deruxtecan (26) (DS-8201a, T-DXd, Enhertu) is a next-generation ADC with a trastuzumab biosimilar conjugated to a novel topoisomerase I inhibitor, deruxtecan, via a hydrophobic, enzymatically cleavable tetrapeptidebased (GGFG) linker with a homogeneous DAR of 8 to 1 (8). In preclinical study, T-DXd was proved to retain the function of trastuzumab post conjugation. The membrane permeable payload grants tumoricidal effect against HER2 heterogeneous tumor via bystander killing effect (8). The pivotal clinical trials of T-DXd were listed in Table 2.

In a pivotal phase II study (DESTINY-Breast01) of HER2 positive MBC patients previously treated with T-DM1, T-DXd demonstrated durable antitumor activities with an impressive objective response rate (ORR) of $60.9 \%$ (95\% CI: $53.4 \%$ to $68.0 \%$ ) and a median PFS of 16.4 months $(95 \%$ 


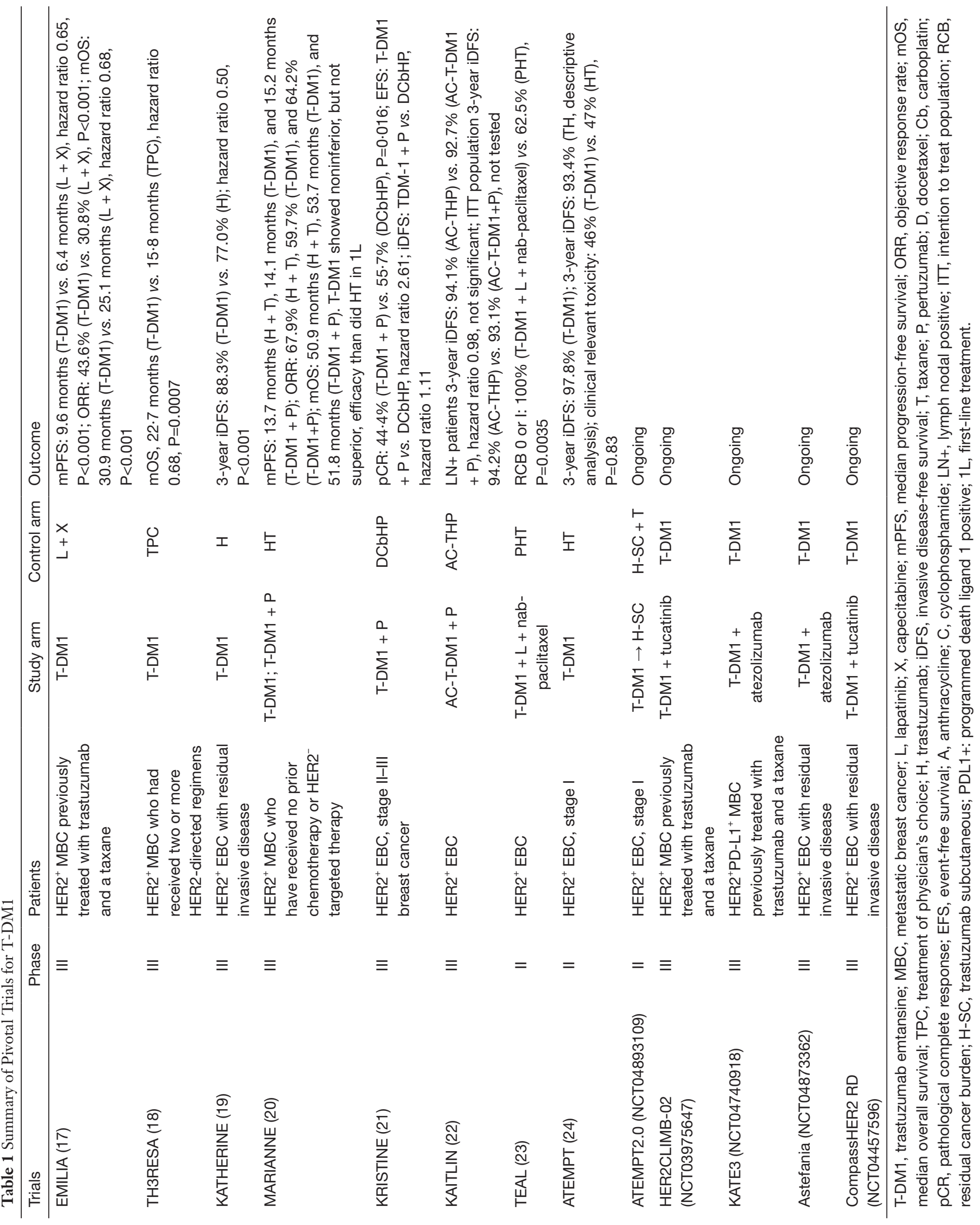


Table 2 Summary of pivotal trials for trastuzumab deruxtecan (T-DXd)

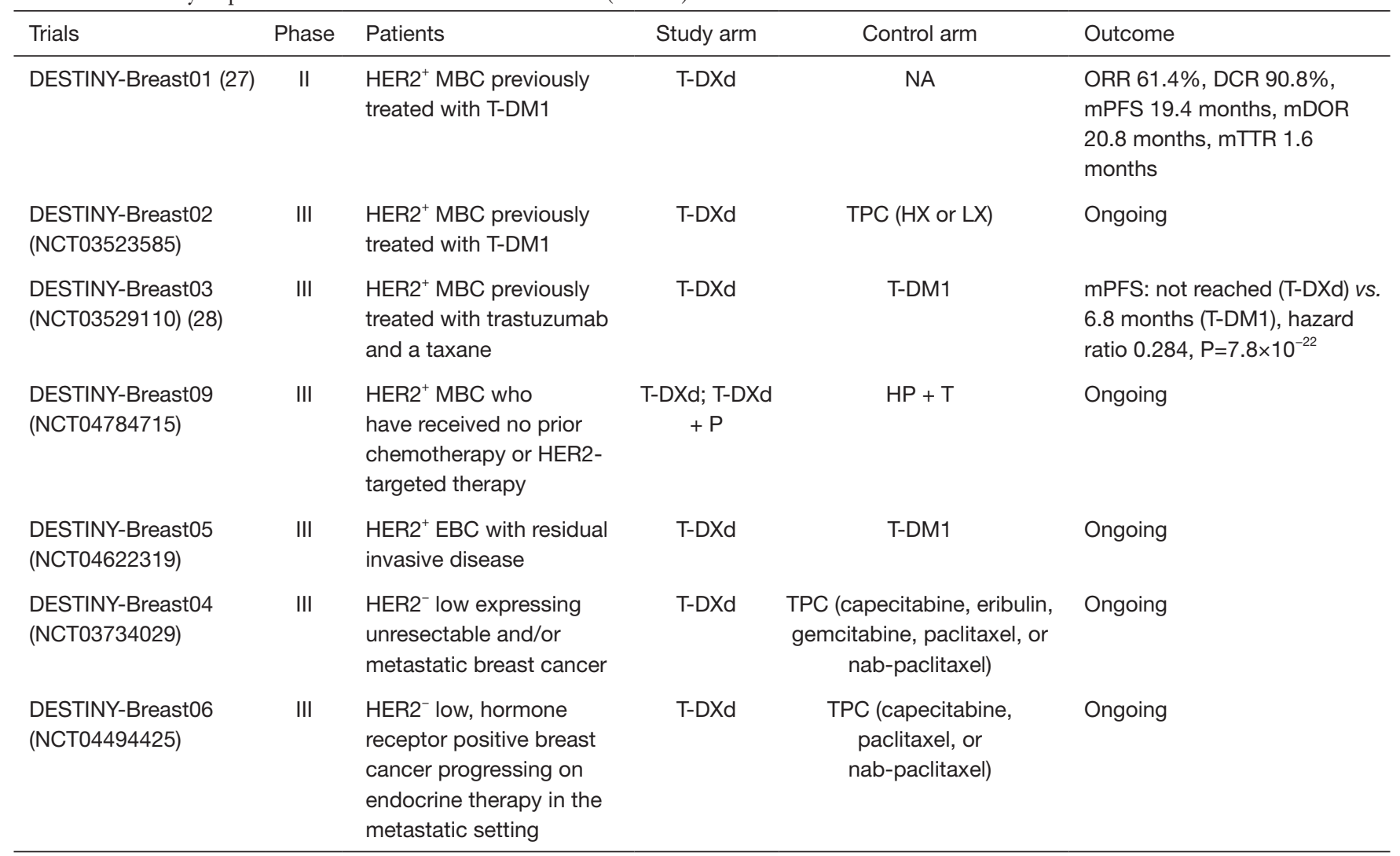

T-DXd, trastuzumab deruxtecan; T-DM1, trastuzumab emtansine; MBC, metastatic breast cancer; ORR, objective response rate; DCR, disease control rate; mDoR, median duration of response; mTTR, median time to response; TPC, treatment of physician's choice; P, pertuzumab; $\mathrm{H}$, trastuzumab; $\mathrm{T}$, taxane.

CI: 12.7 months to not reached) (27). An update result on SABCS 2020 with longer follow-up showed that the PFS and duration of response (DoR) were further extended to 19.4 and 20.8 months, respectively (29). Interestingly, T-DXd seemed to be active in patients with brain metastases, with an ORR of $58.3 \%$ and a median PFS of 18.1 months, similar to the overall population $(30,31)$. Among the patients with information available on baseline brain metastasis diameter, the central nervous system response rate per investigators was $50 \%$ (7 of 14 patients) (30). The phase III DESTINYBreast02 trial (NCT03523585), which is ongoing for evaluating T-DXd $v s$. standard of care after T-DM1 in HER2 positive unresectable and/or MBC with PFS as the primary endpoint, will provide confirmative results.

Moreover, in a recently reported head-to-head comparison study (DESTINY-Breast03, NCT03529110) of T-DXd vs. T-DM1 in patients previously treated with trastuzumab and taxane in the advanced/metastatic setting,
T-DXd demonstrated statistically significant and clinically meaningful improvement in PFS (28). The hazard ratio for PFS was $0.2840\left(\mathrm{P}=7.8 \times 10^{-22}\right)$; median PFS not reached for T-DXd vs. 6.8 months for T-DM1. At the time of the planned interim analysis, a strong OS benefit trend was showed. The estimated 12 -month OS event rates were 94.1\% (95\% CI: $90.3 \%$ to $96.4 \%$ ) for T-DXd and $85.9 \%$ (95\% CI: $80.9 \%$ to $89.7 \%$ ) for T-DM1; hazard ratio: 0.5546 (95\% CI: 0.3587 to 0.8576 ; $\mathrm{P}=0.007172$ did not cross prespecified boundary for significance). It is worth noting that lung toxicity is the critical safety concern related to T-DXd. Comparing to previously reported $2.7 \%$ grade 5 treatment related interstitial lung disease (ILD) (29), there was no grade 4-5 treatment related ILD reported in DESTINYBreast03 trial (28). It seems that higher awareness and earlier line treatment may help reduce the risk of T-DXdrelated ILD. Nevertheless, lung event should be closely monitored and proactively intervened to avoid any 
untoward outcome in clinical practices.

Three factors contribute to the greater efficacy of T-DXd. The conjugate carries a highly membrane permeable payload with strong bystander effect, which enhances the tumoricidal activities of T-DXd against tumor cells with no, weak or moderate HER2 expression $(8,10)$. Furthermore, tumor-specific peptide linker and site-specific conjugation contribute to a homogeneous and high DAR, ensuring a delivery of greater payload to HER2 ${ }^{+}$cells, and a stable PK profile, which helps maintain synergy during long-term treatment. Finally, the function of trastuzumab is not only comprehensively retained in T-DXd at the molecular fusion level but also at the clinical dosage level (27).

There is a continued need for novel treatment for patients with HER2 positive MBC who experience disease progression after initial treatment with standard of care. The results of these trials could transform the standard of care of second-line treatment in HER2 positive MBC. Moreover, in both early and advanced setting of HER2 positive breast cancer, ongoing randomized phase III clinical trials aim at investigating the clinical benefit of T-DXd compared with other approved anti-HER2 treatments.

DESTINY-Breast09 (NCT04784715), a phase III trial, is currently evaluating the efficacy and safety of T-DXd either alone or in combination with pertuzumab vs. firstline standard of care (pertuzumab, trastuzumab and taxane) in patients with HER2 positive breast cancer who have received no prior chemotherapy or HER2-targeted therapy. DESTINY-Breast05 (NCT04622319), a phase III, randomized, open-label study, evaluates the iDFS of T-DXd vs. T-DM1 in patients with high-risk HER2 positive breast cancer with residual invasive disease in breast or axillary lymph nodes in the post-neoadjuvant setting.

This higher DAR of T-DXd has been granted efficacy to deliver more payload to HER2 positive cells, especially for those with weak or moderate HER2 expression (8). Meanwhile, the bystander effect might further enhance the clinical activity even in HER2-low or HER2-heterogenous breast cancers, while ensuring a safe therapeutic index (10). In preclinical trials, T-DXd has demonstrated tumor inhibitory effects in HER2 low $\left(\mathrm{IHC}^{+}{ }^{+}\right.$or $\left.\mathrm{IHC}^{+} / \mathrm{ISH}^{-}\right)$or HER2 positive tumors. J101, a phase Ib study evaluating safety and efficacy of T-DXd in HER2-low expressing MBC, showed that T-DXd achieved an ORR of $37.0 \%$ (95\% CI: $24.3 \%$ to $51.3 \%$ ) with median DoR of 10.4 months in patients that were heavily pretreated (32). Later, HER2 low population has been explored in two phase III trials.

The DESTINY-Breast04 trial (NCT03734029) is currently investigating T-DXd vs. physician's choice (capecitabine, eribulin, gemcitabine, paclitaxel, or nabpaclitaxel) in patients with HER2-low expressing unresectable and/or MBC. Simultaneously, the DESTINYBreast06 (NCT04494425) trial is evaluating the efficacy of T-DXd compared with investigator's choice chemotherapy in HER2-low, hormone receptor (HR) positive breast cancer patients whose disease has progressed on endocrine therapy in the metastatic setting. The results of this study could provide evidence whether T-DXd could provide a novel targeted therapy for advanced HER2-low breast cancer that has progressed on prior lines of therapy.

\section{Sacituzumab govitecan}

Sacituzumab govitecan (SG, Trodelvy) is an anti-TROP-2 ADC. SN-38, which is the active metabolite of the camptothecin, irinotecan and a moderately toxic payload, is conjugated to humanized anti-TROP-2 mAb hRS7 IgG1k via a cleavable and moderately stable linker $(6,7)$. The pivotal trials of SG were summarized in Table 3.

SG was approved for pretreated unresectable locally advanced or metastatic triple-negative breast cancer (mTNBC) based on the phase III ASCENT trial showing significantly longer PFS and OS with SG than single-agent chemotherapy among patients with mTNBC (33). In the exploratory analysis of patients with brain metastases, SG was numerically better than treatment of physician's choice (TPC) for PFS 2.8 months (95\% CI: 1.5 to 3.9 months) for SG vs. 1.6 months (95\% CI: 1.3 to 2.9 months) for TPC, but there were barely no different in terms of ORR (SG $3 \% v s$. TPC $0 \%$ ) or median OS (SG 6.8 months vs. TPC 7.5 months) (36). SG is under further clinical investigation for patients with HER2 negative breast cancer with brain metastasis (NCT04647916).

Additional trials have been conducted to assess SG in other settings for breast cancer (NCT03901339, NCT04595565 and NCT04468061).

In a phase I/II IMMU-132-01 basket study, SG has demonstrated promising activity in heavily pretreated hormonal receptor positive and HER2 negative $\left(\mathrm{HR}^{+} /\right.$ HER2-) MBC (37). Phase III TROPiCS-02 study (NCT03901339) is conduct evaluating SG vs. TPC in $\mathrm{HR}^{+} /$ HER2- MBC. Eligible patients including $\mathrm{HR}^{+} / \mathrm{HER} 2^{-}$ $\mathrm{MBC}$ have been previously treated with at least two but no more than four prior systemic chemotherapy regimens for $\mathrm{MBC}$, including at least one prior taxane in any setting, at least one prior anticancer hormonal treatment and 
Table 3 Summary of pivotal trials for sacituzumab govitecan (SG)

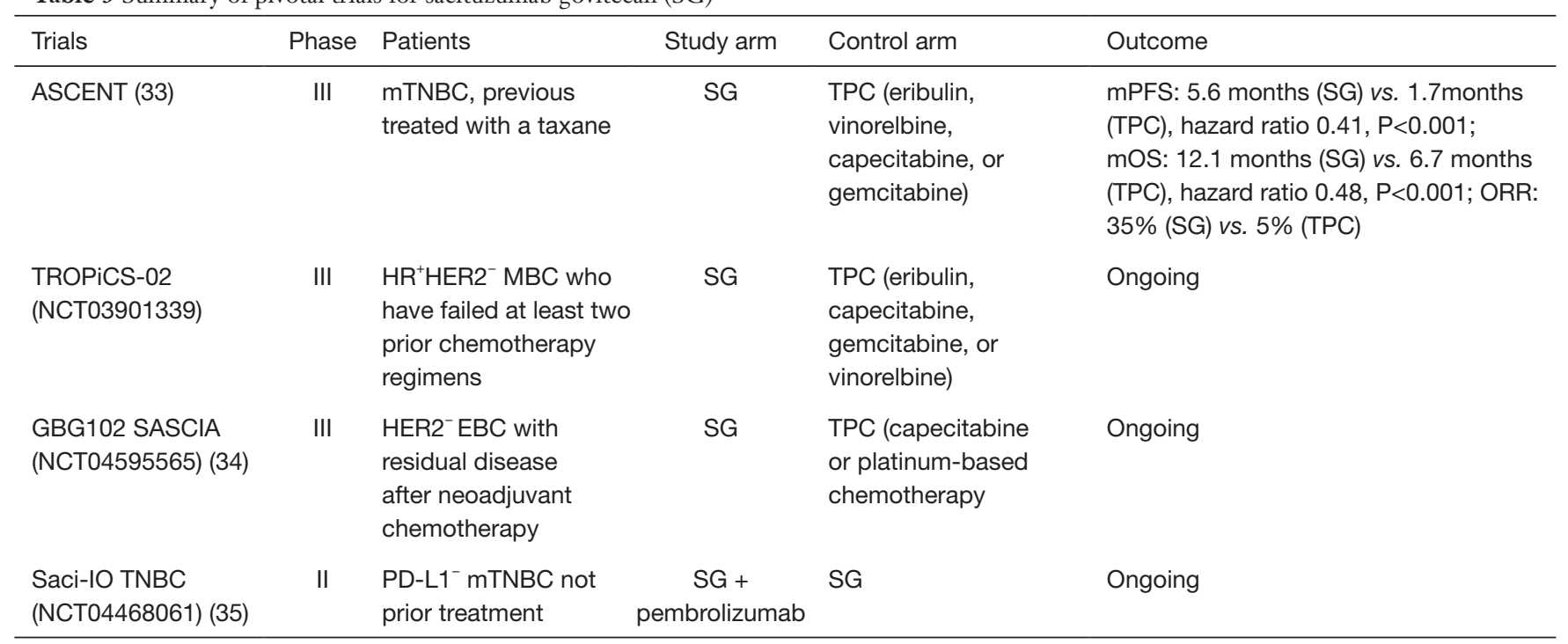

SG, sacituzumab govitecan; mTNBC, metastatic triple negative breast cancer; TPC, treatment of physician's choice; mPFS, median progression-free survival; mOS, median overall survival; ORR, objective response rate; $\mathrm{HR}^{+}$, hormonal receptor positive; HER2- $\mathrm{HER}_{2}$ negative; $\mathrm{PD}-\mathrm{L1}^{-}$, programmed death ligand 1 negative.

at least one CDK4/6 inhibitor in any setting. Patients will be randomized 1:1 to receive $\mathrm{SG}$ or single-agent standard of care TPC (eribulin, capecitabine, gemcitabine or vinorelbine). Primary end points are PFS and overall response rate.

Early HER2 negative patients with residual disease after neoadjuvant chemotherapy (NACT) are presentative population with resistance to initial treatment and at high risk of recurrence. SG may have potential possibility to improve the prognosis of these patients. SASCIA (NCT04595565) is an ongoing phase III study (34), recruiting patients who have residual disease post taxanebased NACT and are at high risk of recurrence, defined as HER2 negative breast cancer, either hormonal receptor negative with any residual invasive disease $>$ ypT1mi after NACT or hormonal receptor positive with a CPS (clinical and post treatment pathological stage) + EG (estrogen receptor status and grade) score $\geq 3$ or CPS + EG score 2 and ypN+. Patients are randomized 1:1 to receive either SG for eight cycles or TPC (capecitabine or platinum-based chemotherapy or observation). The primary endpoint is iDFS.

In preclinical exploration, $\mathrm{SG}$ has shown to have synergic effect with PD-1/PD-L1 antibody (35). Saci-IO TNBC (NCT04468061) is a multi-center 1:1 randomized phase II trial to investigate whether the addition of pembrolizumab to SG improves PFS compared to SG alone in PD-L1 negative
mTNBC with no prior treatment in metastatic setting.

\section{Other promising ADCs under clinical development in breast cancer}

Besides the approved anti-HER2 and anti-TROP-2 ADCs discussed above, several novel ADCs under exploration in breast cancer were listed in Table 4 .

For HER2-targeting ADCs, the data from clinical trials is telling different stories. BAT8001 (38) is an ADC targeting HER2 positive cells composed of a trastuzumab biosimilar linked to the drug-linker Batansine, which has a structure somewhat similar to T-DM1. However, its phase III study failed to meet the superior primary endpoint of PFS when comparing BAT8001 vs. lapatinib plus capecitabine in HER2 positive MBC progressing on trastuzumab and taxane regimen (39). Once again, it showed the limitation of last generation ADCs. Disitamab vedotin (RC48) is an ADC composed of an anti-HER2 humanized mAb (disitamab) coupled to average four molecules (DAR, 4:1) of monomethyl auristatin $E$ warhead via a protease cleavable linker (40). RC48 has been approved for HER2 positive gastric cancer patients who have been treated with previous two lines of chemotherapy (49). Recently, a pooled analysis of two phase I trials (NCT02881138 and NCT03052634) showed promising activities of RC48-ADC in HER2 positive and HER2-low breast cancer (40). Several phase II/III trials 
Table 4 Selective ongoing trials for novel ADCs in breast cancer

\begin{tabular}{|c|c|c|c|c|c|}
\hline Trials & Phase & Patients & Study arm & Control arm & Outcome \\
\hline \multicolumn{6}{|l|}{ Trials of anti-HER2 ADCs } \\
\hline $\begin{array}{l}\text { BAT-8001-001-CR } \\
\text { (NCT04189211) (38) }\end{array}$ & 1 & $\mathrm{HER}^{+} \mathrm{MBC}$ & BAT8001 & - & $\begin{array}{l}\text { ORR: } 41.4 \%, 95 \% \mathrm{Cl}: 23.5 \% \text { to } \\
61.1 \%) \text {; DCR: } 82.8 \% \text {, } 95 \% \mathrm{Cl}: 64.2 \% \\
\text { to } 94.2 \%\end{array}$ \\
\hline $\begin{array}{l}\text { BAT-8001-002-CR } \\
\text { (NCT04185649) (39) }\end{array}$ & III & $\begin{array}{l}\mathrm{HER} 2^{+} \mathrm{MBC} \text { with } \\
\text { previous progression } \\
\text { on } \mathrm{H}\end{array}$ & BAT8001 & $L+X$ & Reported as failed \\
\hline $\begin{array}{l}\text { C001 CANCER } \\
\text { (NCT02881138); C003 } \\
\text { CANCER (NCT03052634) (40) }\end{array}$ & I-IB & $\begin{array}{l}\mathrm{HER}^{+} \text {and HER2 low } \\
\mathrm{MBC}\end{array}$ & $\mathrm{RC} 48$ & NA & $\begin{array}{l}\text { HER2-positive subgroup } 2.0 \mathrm{mg} / \mathrm{kg} \\
\text { q2w (RD for phase III), ORR } 42.9 \% \\
\text { ( } 9 / 21) \text {, mPFS } 5.7 \text { months; HER2-low } \\
\text { subgroup, ORR } 39.6 \% \text { (19/48); mPFS } \\
5.7 \text { months }\end{array}$ \\
\hline RC48-C012 (NCT04400695) & III & $\begin{array}{l}\text { HER2-low MBC } \\
\text { with 1-2L previous } \\
\text { chemotherapy }\end{array}$ & RC48 & $\begin{array}{l}\text { Physician's } \\
\text { choice }\end{array}$ & Ongoing \\
\hline $\begin{array}{l}\text { SYD985.001 (NCT02277717) } \\
(41)\end{array}$ & 1 & $\begin{array}{l}\text { HER2 }{ }^{+} \mathrm{MBC} \text { with } \\
\text { previous progression } \\
\text { on SOC }\end{array}$ & SYD985 & NA & $\begin{array}{l}\text { HER2-positive group, ORR 33\% } \\
\text { (16/48), mPFS 9.4 months; HER2-low/ } \\
\text { HR-positive disease, ORR } 27 \%(8 / 30) \text {, } \\
\text { mPFS } 4.1 \text { months; HER2-low/TNBC, } \\
\text { ORR 40\% (6/15), mPFS } 4.4 \text { months }\end{array}$ \\
\hline TULIP (NCT03262935) (42) & III & $\mathrm{HER}^{+} \mathrm{MBC} \geq 2 \mathrm{~L}$ & SYD985 & $\begin{array}{l}\text { Physician's } \\
\text { choice }\end{array}$ & $\begin{array}{l}\text { mPFS: } 7.0 \text { months (SYD985) vs. } \\
4.9 \text { months (Physician's choice), } \\
\text { hazard ratio } 0.64, \mathrm{P}=0.002\end{array}$ \\
\hline $\begin{array}{l}\text { KL166-I-01-CTP } \\
(\text { CTR20181301) (45) }\end{array}$ & 1 & $\begin{array}{l}\text { HER2 }{ }^{+} \text {locally } \\
\text { advanced or } \\
\text { metastatic solid } \\
\text { tumors }\end{array}$ & A166 & NA & $\begin{array}{l}\text { ORR: } 59.1 \%(13 / 22) 4.8 \mathrm{mg} / \mathrm{kg} \text { cohort; } \\
71.4 \%(10 / 14) 6.0 \mathrm{mg} / \mathrm{kg} \text { cohort }\end{array}$ \\
\hline KL166-II-02 (CTR20212088) & II & $\mathrm{HER}^{+} \mathrm{MBC} \geq 2 \mathrm{~L}$ & A166 & NA & Ongoing, A166 with $4.8 \mathrm{mg} / \mathrm{kg}$ q3w \\
\hline \multicolumn{6}{|l|}{ Trials of ADCs with other targeting } \\
\hline $\begin{array}{l}\text { TROPION-PanTumor01 } \\
\text { (NCT03401385) (46) }\end{array}$ & 1 & $\begin{array}{l}\text { mTNBC with previous } \\
\text { progression on SOC }\end{array}$ & Dato-DXd & NA & $\begin{array}{l}\text { mTNBC cohort: ORR 43\% (9/21), DCR } \\
95 \%(20 / 21)\end{array}$ \\
\hline $\begin{array}{l}\text { U31402-A-J101 } \\
\text { (NCT02980341) (47) }\end{array}$ & $\mathrm{I} / \mathrm{II}$ & $\begin{array}{l}\text { HER3-expressing } \\
\text { mTNBC }\end{array}$ & HER3-DXd & NA & $\begin{array}{l}\text { HER3-expressing MBC, ORR 33\% } \\
\text { (7/21), DCR 95\% (20/21) }\end{array}$ \\
\hline $\begin{array}{l}\text { SGNLVA-002 (NCT03310957) } \\
\text { (48) }\end{array}$ & IB/II & $\begin{array}{l}\text { mTNBC without } \\
\text { previous treatment }\end{array}$ & $\begin{array}{l}\text { Ladiratuzumab } \\
\text { vedotin (SGN- } \\
\text { LIV1A) }\end{array}$ & NA & ORR $54 \%(14 / 26)$ \\
\hline
\end{tabular}

MBC, metastatic breast cancer; ORR, objective response rate; $\mathrm{Cl}$, confidence interval; DCR, disease control rate; L, lapatinib; $\mathrm{X}$, capecitabine; H, trastuzumab; RC48, disitamab vedotin; RD, recommended dose; mPFS, median progression-free survival; SYD985, trastuzumab duocarmazine; mTNBC, metastatic triple negative breast cancer; Dato-DXd, datopotamab deruxtecan or DS-1062; SOC, standard of care; HER3-DXd, patritumab deruxtecan or U3-1402; NA, not available; 1L, first-line treatment; 2L, second-line treatment. 
are currently ongoing to explore the efficacy and safety of RC48-ADC in advanced breast cancer (NCT03500380; NCT04400695). The preliminary outcomes for other ADCs have also reported recently (41-45).

Datopotamab deruxtecan (Dato-DXd, DS-1062) is an ADC comprising a humanized anti-TROP-2 IgG1 mAb conjugated to a potent topoisomerase I inhibitor payload (DXd) via a stable tetrapeptide-based cleavable linker (46). Preliminary results from the mTNBC cohort in TROPION-PanTumor01 study showed that Dato-DXd had highly encouraging antitumor activity, ORR of $43 \%$ and DCR of $95 \%$, and a manageable safety profile in pts with refractory $\mathrm{m}$ TNBC.

There are several promising ADCs targeting different antigens are also under exploring in breast cancer $(47,48)$.

\section{Future prospects}

The success of these novel ADCs has transformed breast cancer treatment. T-DM1 has shown its value in breast cancer treatment, either as palliative therapy in metastatic or escalation therapy for cure in adjuvant setting. The unprecedented efficacy of T-DXd has been promptly translated to clinical usage and the drug conjugate is being explored in earlier setting (first-line treatment and neoadjuvant/adjuvant treatment) and challenges the position of pertuzumab and trastuzumab regimen. Meanwhile, the promising efficacy of T-DXd in HER2 low breast cancer may be proved in confirmative trials and set a new age for treatment of HER2 low breast cancer. Numerous novel ADCs have been under development targeting TROP2 and other antigens in breast cancer and show promising efficacy in clinical trials. We are embracing a new era of ADCs in breast cancer treatment, which will provide more treatment opportunities and be translated to clinical benefits for breast cancer patients.

\section{Acknowledgments}

Funding: None.

\section{Footnote}

Reporting Checklist: The authors have completed the Narrative Review reporting checklist. Available at https:// dx.doi.org/10.21037/tbcr-21-30

Conflicts of Interest: Both authors have completed the
ICMJE uniform disclosure form (available at https://dx.doi. org/10.21037/tbcr-21-30). CH serves as an unpaid editorial board member of Translational Breast Cancer Research. The other author has no conflicts of interest to declare.

Ethical Statement: The authors are accountable for all aspects of the work in ensuring that questions related to the accuracy or integrity of any part of the work are appropriately investigated and resolved.

Open Access Statement: This is an Open Access article distributed in accordance with the Creative Commons Attribution-NonCommercial-NoDerivs 4.0 International License (CC BY-NC-ND 4.0), which permits the noncommercial replication and distribution of the article with the strict proviso that no changes or edits are made and the original work is properly cited (including links to both the formal publication through the relevant DOI and the license). See: https://creativecommons.org/ licenses/by-nc-nd/4.0/.

\section{References}

1. Schwartz RS. Paul Ehrlich's magic bullets. N Engl J Med 2004;350:1079-80.

2. Ford CH, Newman CE, Johnson JR, et al. Localisation and toxicity study of a vindesine-anti-CEA conjugate in patients with advanced cancer. Br J Cancer 1983;47:35-42.

3. Tsuchikama K, An Z. Antibody-drug conjugates: recent advances in conjugation and linker chemistries. Protein Cell 2018;9:33-46.

4. Drago JZ, Modi S, Chandarlapaty S. Unlocking the potential of antibody-drug conjugates for cancer therapy. Nat Rev Clin Oncol 2021;18:327-44.

5. Sun X, Ponte JF, Yoder NC, et al. Effects of DrugAntibody Ratio on Pharmacokinetics, Biodistribution, Efficacy, and Tolerability of Antibody-Maytansinoid Conjugates. Bioconjug Chem 2017;28:1371-81.

6. Starodub AN, Ocean AJ, Shah MA, et al. First-inHuman Trial of a Novel Anti-Trop-2 Antibody-SN-38 Conjugate, Sacituzumab Govitecan, for the Treatment of Diverse Metastatic Solid Tumors. Clin Cancer Res 2015;21:3870-8.

7. Goldenberg DM, Sharkey RM. Antibody-drug conjugates targeting TROP-2 and incorporating SN-38: A case study of anti-TROP-2 sacituzumab govitecan. MAbs 2019;11:987-95.

8. Ogitani Y, Aida T, Hagihara K, et al. DS-8201a, A Novel 
HER2-Targeting ADC with a Novel DNA Topoisomerase I Inhibitor, Demonstrates a Promising Antitumor Efficacy with Differentiation from T-DM1. Clin Cancer Res 2016;22:5097-108.

9. Junttila TT, Li G, Parsons K, et al. TrastuzumabDM1 (T-DM1) retains all the mechanisms of action of trastuzumab and efficiently inhibits growth of lapatinib insensitive breast cancer. Breast Cancer Res Treat 2011;128:347-56.

10. Ogitani $Y$, Hagihara $K$, Oitate $M$, et al. Bystander killing effect of DS-8201a, a novel anti-human epidermal growth factor receptor 2 antibody-drug conjugate, in tumors with human epidermal growth factor receptor 2 heterogeneity. Cancer Sci 2016;107:1039-46.

11. Gonzalez-Angulo AM, Litton JK, Broglio KR, et al. High risk of recurrence for patients with breast cancer who have human epidermal growth factor receptor 2-positive, node-negative tumors $1 \mathrm{~cm}$ or smaller. J Clin Oncol 2009;27:5700-6.

12. Onitilo AA, Engel JM, Greenlee RT, et al. Breast cancer subtypes based on ER/PR and Her2 expression: comparison of clinicopathologic features and survival. Clin Med Res 2009;7:4-13.

13. Slamon DJ, Clark GM, Wong SG, et al. Human breast cancer: correlation of relapse and survival with amplification of the HER-2/neu oncogene. Science 1987;235:177-82.

14. Keam SJ. Trastuzumab Deruxtecan: First Approval. Drugs 2020;80:501-8.

15. Goldenberg DM, Cardillo TM, Govindan SV, et al. Trop-2 is a novel target for solid cancer therapy with sacituzumab govitecan (IMMU-132), an antibody-drug conjugate (ADC). Oncotarget 2015;6:22496-512.

16. Lewis Phillips GD, Li G, Dugger DL, et al. Targeting HER2-positive breast cancer with trastuzumab-DM1, an antibody-cytotoxic drug conjugate. Cancer Res 2008;68:9280-90.

17. Verma S, Miles D, Gianni L, et al. Trastuzumab emtansine for HER2-positive advanced breast cancer. N Engl J Med 2012;367:1783-91.

18. Krop IE, Kim SB, Martin AG, et al. Trastuzumab emtansine versus treatment of physician's choice in patients with previously treated HER2-positive metastatic breast cancer (TH3RESA): final overall survival results from a randomised open-label phase 3 trial. Lancet Oncol 2017;18:743-54.

19. von Minckwitz G, Huang CS, Mano MS, et al. Trastuzumab Emtansine for Residual Invasive HER2-
Positive Breast Cancer. N Engl J Med 2019;380:617-28.

20. Perez EA, Barrios C, Eiermann W, et al. Trastuzumab Emtansine With or Without Pertuzumab Versus Trastuzumab Plus Taxane for Human Epidermal Growth Factor Receptor 2-Positive, Advanced Breast Cancer: Primary Results From the Phase III MARIANNE Study. J Clin Oncol 2017;35:141-8. Erratum in: J Clin Oncol. 2017 Jul 10;35(20):2342. J Clin Oncol. 2019 Feb 1;37(4):358

21. Hurvitz SA, Martin M, Symmans WF, et al. Neoadjuvant trastuzumab, pertuzumab, and chemotherapy versus trastuzumab emtansine plus pertuzumab in patients with HER2-positive breast cancer (KRISTINE): a randomised, open-label, multicentre, phase 3 trial. Lancet Oncol 2018;19:115-26.

22. Harbeck N, Im SA, Barrios CH, et al. Primary analysis of KAITLIN: A phase III study of trastuzumab emtansine (T-DM1) + pertuzumab versus trastuzumab + pertuzumab + taxane, after anthracyclines as adjuvant therapy for highrisk HER2-positive early breast cancer (EBC). J Clin Oncol 2020;38:abstr 500.

23. Patel TA, Ensor JE, Creamer SL, et al. A randomized, controlled phase II trial of neoadjuvant ado-trastuzumab emtansine, lapatinib, and nab-paclitaxel versus trastuzumab, pertuzumab, and paclitaxel in HER2positive breast cancer (TEAL study). Breast Cancer Res 2019;21:100.

24. Tolaney SM, Tayob N, Dang C, et al. Adjuvant Trastuzumab Emtansine Versus Paclitaxel in Combination With Trastuzumab for Stage I HER2-Positive Breast Cancer (ATEMPT): A Randomized Clinical Trial. J Clin Oncol 2021;39:2375-85.

25. Ruddy KJ, Zheng Y, Tayob N, et al. Chemotherapyrelated amenorrhea (CRA) after adjuvant ado-trastuzumab emtansine (T-DM1) compared to paclitaxel in combination with trastuzumab (TH) (TBCRC033: ATEMPT Trial). Breast Cancer Res Treat 2021;189:103-10.

26. Nakada T, Sugihara K, Jikoh T, et al. The Latest Research and Development into the Antibody-Drug Conjugate, fam- Trastuzumab Deruxtecan (DS-8201a), for HER2 Cancer Therapy. Chem Pharm Bull (Tokyo) 2019;67:173-85.

27. Modi S, Saura C, Yamashita T, et al. Trastuzumab Deruxtecan in Previously Treated HER2-Positive Breast Cancer. N Engl J Med 2020;382:610-21.

28. Cortés J, Kim SB, Chung WP, et al. LBA1 - Trastuzumab deruxtecan (T-DXd) vs trastuzumab emtansine (T-DM1) in patients (Pts) with HER2+ metastatic breast cancer 
(mBC): Results of the randomized phase III DESTINYBreast03 study. Ann Oncol 2021;32:S1287-8.

29. Modi S, Saura C, Yamashita T, et al. Updated results from DESTINY-breast01, a phase 2 trial of trastuzumab deruxtecan (T-DXd ) in HER2 positive metastatic breast cancer. Cancer Res 2021;81:abstr PD3-06.

30. Jerusalem GHM, Park YH, Yamashita T, et al. Trastuzumab deruxtecan (T-DXd) in patients with HER2 + metastatic breast cancer with brain metastases: A subgroup analysis of the DESTINY-Breast01 trial. J Clin Oncol 2021;39:abstr 526.

31. Jerusalem G, Park YH, Yamashita T, et al. 138 O CNS metastases in HER2-positive metastatic breast cancer treated with trastuzumab deruxtecan: DESTINY-Breast01 subgroup analyses. Ann Oncol 2020;31:S63-S4.

32. Modi S, Park H, Murthy RK, et al. Antitumor Activity and Safety of Trastuzumab Deruxtecan in Patients With HER2-Low-Expressing Advanced Breast Cancer: Results From a Phase Ib Study. J Clin Oncol 2020;38:1887-96.

33. Bardia A, Hurvitz SA, Tolaney SM, et al. Sacituzumab Govitecan in Metastatic Triple-Negative Breast Cancer. N Engl J Med 2021;384:1529-41.

34. Marmé F, Stickeler E, Furlanetto J, et al. Phase III postneoadjuvant study evaluating sacituzumab govitecan, an antibody drug conjugate in primary HER2-negative breast cancer patients with high relapse risk after standard neoadjuvant treatment: SASCIA. J Clin Oncol 2021;39:abstr TPS602.

35. Garrido-Castro AC, Keenan TE, Li T, et al. SaciIO TNBC: Randomized phase II trial of sacituzumab govitecan (SG) +/- pembrolizumab in PD-L1- metastatic triple-negative breast cancer (mTNBC). J Clin Oncol 2021;39:abstr TPS1106.

36. Diéras V, Weaver R, Tolaney SM, et al. Subgroup analysis of patients with brain metastases from the phase 3 ASCENT study of sacituzumab govitecan versus chemotherapy in metastatic triple-negative breast cancer. Cancer Res 2021;81:abstr PD13-07.

37. Kalinsky K, Diamond JR, Vahdat LT, et al. Sacituzumab govitecan in previously treated hormone receptor-positive/ HER2-negative metastatic breast cancer: final results from a phase I/II, single-arm, basket trial. Ann Oncol 2020;31:1709-18.

38. Hong R, Xia W, Wang L, et al. Safety, tolerability, and pharmacokinetics of BAT8001 in patients with HER2 positive breast cancer: An open-label, dose-escalation, phase I study. Cancer Commun (Lond) 2021;41:171-82.

39. Bio-Thera Solutions, Ltd. Voluntary disclosure of informative announcements regarding the progress of BAT8001 clinical trials. Available online: https://www.biothera.com/uploads/soft/210208/3-21020Q61357.pdf

40. Wang J, Liu Y, Zhang Q, et al. RC48-ADC, a HER2targeting antibody-drug conjugate, in patients with HER2positive and HER2-low expressing advanced or metastatic breast cancer: A pooled analysis of two studies. J Clin Oncol 2021;39:abstr 1022.

41. Banerji U, van Herpen CML, Saura C, et al. Trastuzumab duocarmazine in locally advanced and metastatic solid tumours and HER2-expressing breast cancer: a phase 1 dose-escalation and dose-expansion study. Lancet Oncol 2019;20:1124-35.

42. Manich CS, O'Shaughnessy J, Aftimos PG, et al. LBA15 - Primary outcome of the phase III SYD985.002/TULIP trial comparing vic- trastuzumab duocarmazine to physician's choice treatment in patients with pre-treated HER2-positive locally advanced or metastatic breast cancer. Ann Oncol 2021;32:S1288.

43. Hu X, Zhang J, Ji D, et al. A phase 1 study of ARX788, a HER2-targeting antibody-drug conjugate, in patients with metastatic HER2-positive breast cancer. Cancer Res 2020;80:abstr P1-18-6.

44. Hu X, Zhang J, Wang L, et al. A randomized, multicenter, open-label phase II/III study of ARX788 vs Lapatinib and Capecitabine in patients with HER2positive locally advanced or metastatic breast cancer (ZMC-ARX788-211). Cancer Res 2021;81:abstr PS1057-PS10-57.

45. Hu X, Zhang J, Liu R, et al. Phase I study of A166 in patients with HER2-expressing locally advanced or metastatic solid tumors. J Clin Oncol 2021;39:abstr 1024.

46. Bardia A. LBA4 - Datopotamab deruxtecan (Dato-DXd), a TROP2-directed antibody-drug conjugate (ADC), for triple-negative breast cancer (TNBC): Preliminary results from an ongoing phase 1 trial. Ann Oncol 2021;32:S60-S78.

47. Kogawa T, Yonemori K, Masuda N, et al. Single agent activity of U3-1402, a HER3-targeting antibodydrug conjugate, in breast cancer patients: Phase 1 dose escalation study. J Clin Oncol 2018;36:abstr 2512.

48. Han H, Diab S, Alemany C, et al. Open label phase 1b/2 study of ladiratuzumab vedotin in combination with pembrolizumab for first-line treatment of patients with unresectable locally-advanced or metastatic triple-negative breast cancer. Cancer Res 2020;80:abstr PD1-06.

49. ADC Review Editorial Team. Chinese Regulator Conditionally Approves Disitamab Vedotin in Advanced 
or Metastatic Gastric Cancer. Available online: https:// www.adcreview.com/clinical-trials-update/chineseregulator-conditionally-approves-disitamab-vedotin-in- advanced-or-metastatic-gastric-cancer/ (accessed 15th September 2021).

doi: $10.21037 /$ tbcr-21-30

Cite this article as: Hao C, Liu H. Current understandings and prospects of antibody-drug conjugates (ADCs) for the treatment of breast cancer: a narrative review. Transl Breast Cancer Res 2021;2:30. 